Témoigner Témoigner. Entre histoire et mémoire

Getuigen Revue pluridisciplinaire de la Fondation Auschwitz

$123 \mid 2016$

Traduire le témoignage

\title{
Guy Cassiers et Erwin Jans, Les bienveillantes
}

De Welwillenden

\section{Anneleen Spiessens}

\section{(2) OpenEdition}

\section{Journals}

Édition électronique

URL : https://journals.openedition.org/temoigner/5059

DOI : 10.4000/temoigner.5059

ISSN : 2506-6390

Éditeur :

Éditions du Centre d'études et de documentation Mémoire d'Auschwitz, Éditions Kimé

Édition imprimée

Date de publication : 1 octobre 2016

Pagination : 17-21

ISBN : 987 2-9600926-4-6

ISSN : 2031-4183

Référence électronique

Anneleen Spiessens, "Guy Cassiers et Erwin Jans, Les bienveillantes», Témoigner. Entre histoire et mémoire [En ligne], 123 | 2016, mis en ligne le 02 novembre 2021, consulté le 04 novembre 2021. URL : http://journals.openedition.org/temoigner/5059; DOI : https://doi.org/10.4000/temoigner.5059 


\section{LES BIENVEILLANTES}

THEATRE «Laissez-moi vous raconter comment ça s'est passé. Il s'agit d'une sombre histoire, mais édifiante aussi, un conte moral disons. Ce sera assez long, après tout il s'est passé beaucoup de choses. Mais si ça se trouve vous n'êtes pas trop pressés, peut-être avez-vous le temps. Je ferai de mon mieux pour rester clair. Je peux vous assurer que mon récit sera libre de toute contrition. Je ne regrette rien, j’ai fait mon travail, voilà tout. »

C'est Max Aue, incarné par l'acteur néerlandais Hans Kesting, qui prend la parole. Vers la fin de sa vie, il passe en revue sa carrière auprès du SD, puis de la SS, où il a marché dans le sillage des Einsatzgruppen allemands et surveillé la «Shoah par balles » au front de l'Est. Après un passage à Stalingrad, il est détaché à Berlin pour étudier la contribution des Juifs emprisonnés pour l'effort de guerre. Depuis l'avant-scène, il s'adresse directement à son public.

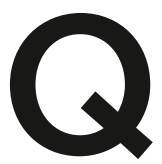
uand Jonathan Littell, écrivain américain alors inconnu, publia en 2006 Les Bienveillantes, il rencontra un succès éditorial instantané. Il fut vite traduit en plus de quinze langues et se vit en outre attribuer le Prix Goncourt et le Prix de l'Académie française. Pourtant, une partie de la critique française (et américaine) descendit le livre en flammes, dénonçant l'invraisemblable et inconcevable mélange de fiction et d'histoire et se scandalisant de la perspective narrative choisie, qui est celle du nazi ${ }^{1}$. Voilà précisément les deux aspects - le rôle de la fiction et la narration du bourreau - que Guy Cassiers (metteur en scène) et Erwin Jans (dramaturge) ont voulu interroger dans leur adaptation du roman.

\section{L'IMAGINATION ET LA SHOAH}

La conception de la scène est minimaliste: point de croix gammées, d'uniformes SS ou de drapeaux rouges, mais par contre des armoires à archives, rouillées et entassées dans un décor stérile. Cassiers et Jans se sont visiblement inspirés des installations de Christian Boltanski, construites autour de vieux documents, de collections et d'archives pour symboliser la lutte contre l'oubli. Une lutte qui s'avère personnelle pour l'artiste : Boltanski est né le jour où Paris est libéré, ce qui le fait appartenir à la génération « postmémorielle » (Hirsch, 1996, p. 675) sans souvenirs propres de la Shoah. «My work is not about, it is after », confirme-t-il lors d'un entretien avec Georgia Marsh (Marsh, 1990, p. 10). C'est par des «effets Shoah» (van Alphen, 2007, p. 368), à l'aide de références symboliques et non littérales donc, que Boltanski évoque les événements du passé ainsi que l'idée de distance, d'absence et de perte qui y est associée. Les archives sur la scène à Anvers ne renvoient qu'implicitement à la Shoah et constituent en premier lieu une métaphore de la mémoire (absente). Si l'armoire est fermée dans la première partie du spectacle, elle semble prendre vie dans la deuxième : l'un après l'autre, les casiers s'ouvrent automatiquement, transformant les archives en un monstre gigantesque. «D'un coup je ressens tout le poids du passé, constate Aue, de la douleur de la vie et de la mémoire inaltérable.»

Les archives ne représentent pas seulement la mémoire collective et personnelle; elles renvoient $\bullet \bullet$

(1) Les Bienveillantes se place pourtant dans une tradition littéraire qui combine la narration du bourreau (ou la focalisation interne) à la mise en scène romanesque ou même magique. Pensons à L'Enfance d'un chef (Sartre, 1939), Deutsches Requiem (Borges, 1946), Le Roi des Aulnes (Tournier, 1970), Le Nazi et le barbier (Hilsenrath, 1971), Le Bonheur nazi (Rachline, 1972). En anglais et en néerlandais : The Man in the High Castle (Dick, 1962), The Iron Dream (Spinrad, 1972), Siegfried (Mulisch, 2001) ou The Castle in the Forest (Mailer, 2007). Pour une analyse approfondie de la perspective du bourreau en littérature, voir Albert Mingelgrün, « La figure du bourreau nazi au tournant du XXle siècle. Quelques variations littéraires », 2009, et Anneleen Spiessens, Quand le bourreau prend la parole : témoignage et fiction, 2016. 


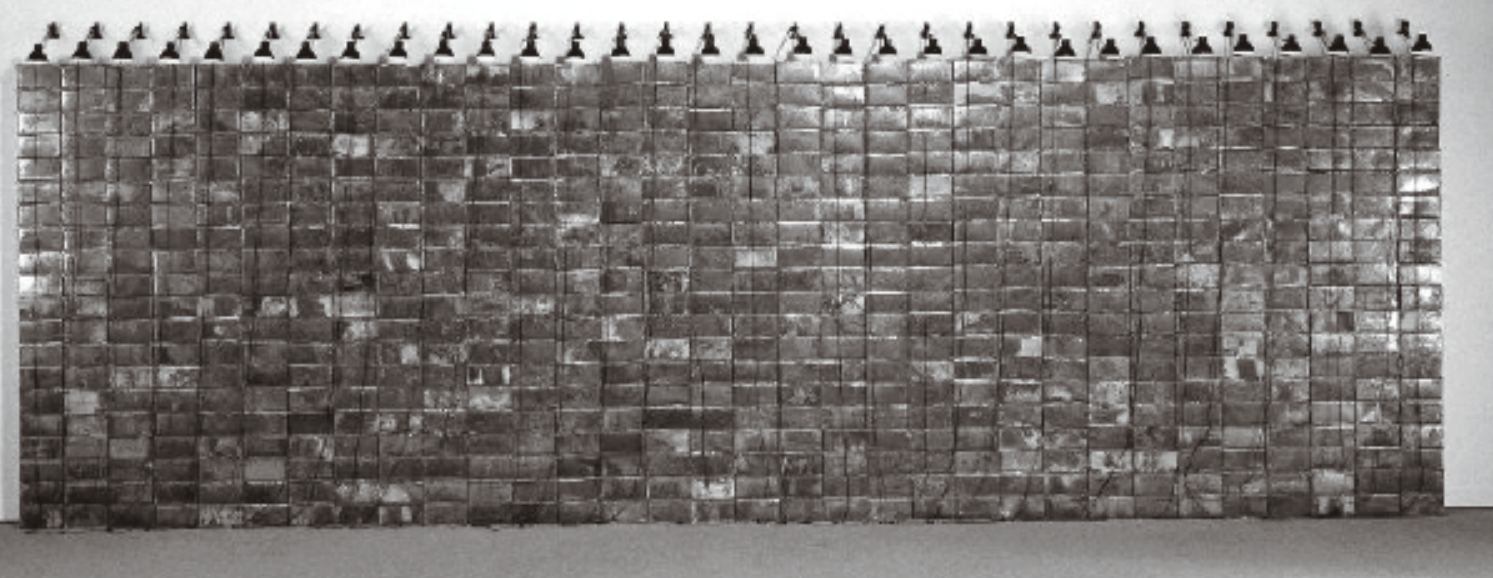

_Les Archives de C.B. Installation de 646 boîtes à biscuits contenant environ 1200 photographies et 800 documents divers.

• aussi à la «méthode» si particulière qui a caractérisé Shoah. En effet, les nazis inventoriaient et répe enlever toute forme d'ins victimes, au point de leur

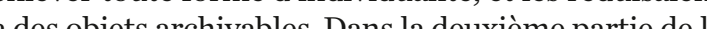
à l'ats archivables. Dans la deuxième partie de la pièce, larmoire à archives sur scène est rècuperee par pichmann, interprété par Katelijne Damen comme un personagepetit-bourgeoisetnér let que. Elles forment . Jons ternes entre bureaucrates allemands où chaque

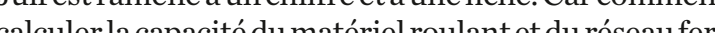
calculer lacapaitedumatén che paire mouri

Dans ce sens, les archives de Boltanski - ainsi que décor de Cassiers - renferment une critique de la le decor de Cassiers - renferment une critique de la lévénemation pures lillusion d'un accès dire il à un pá a procurent lillusion d'un accès direct à un passe authentique hais masquent souvent les processus de sélection et coltanski par rapport au docus Bótanski par rapport aú document, qui ne révèle pas mécanism intlectuels de l'archivage qui sont pré-

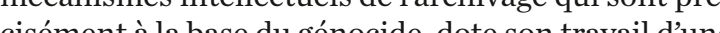
dimension for duct dimension fondamentalement ronique (voir BergBoltari, 2 assers revendique le drot de la fition, du théâtre en l'occurrence, de traiter le sujet délicat du judéocide selon ses procédés propres et en puisant dans son langage visuel specifique. Cest par la voie de la fiction, nus inc ique le netteur en scene, qu'on arrive a associer une intensite émotionnelle aux faits historéalité (Pent \& Cassies, 2015, p. 64-68). lité (Perrot \& Cassiers, 2015, p. 64-68)

Le langage visuel de Cassiers et Jans contient clairement une réflexion sur le pouvoir mais auss l'impuisant unexion sur le pouvoir mais aussi tellement exposés, quotidiennement àune sonnes d'images exposant la cruauté partout an surcharge la destruction, et la souffrance», soutient Cassiers mémoire. Mais le problème avec les imeses fore de les voir, ons'y avec les inages cest qua 2015, p. 64) Mis à part la voie ferroviaire, la ccénographie évite phie evite ace ciconspection les inages clichès que nous associons à la Shoah. Au lieu dintroduire de labstraction et la poésie. Le massacre de Babi Yar, ou les nazis tuent plus de 33000 Juifs en deux jours, est ainsivisualise al laide dune installation qui est montée

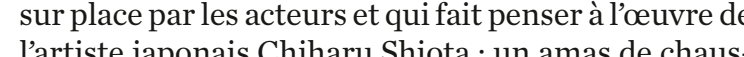
lartiste japonais Chiharu Shiota : un amas de chaussures suspendues au-dessus de la scène avec de long du dans une interview dans Témoigner Entre histoire réferences littérales a l époque nazie, Cassiers préfere physique du disparu. Il est évident que la représentation de laviolence extrême est une question hautement délicaters tabous (penson aux propos de Claude Lanzmann cue la fiction, et le thétre en particulier, disposent que la fiction, et le théatre en particulier, disposent historique autant que d'une responsabilité éthique.

\section{MOI, LE BOURREAU NAZI}

L’officier nazi créé par Cassiers et Kesting n'est pas un psychopathe. Certes, il est hanté par «la passion de d'aventure et du radical à une époque où l'État d'aventure et du radicá à une époque où létat allemand a lui-même lancé un projet radical, le mène

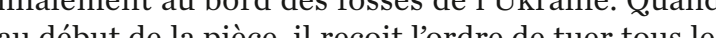
Juifs - fét la piéce, il reşoit lordre de tuer tous les doute Bien que ce doute soit in doute. Bien que ce doute soit inspire par un argument ration au (cestion de la poption cace au problene de la "gestion de la population»), Plus tard, il engage une converstion avec le pat Yakov, un garçon juif qui joue du Bach avec le petit akov, un (ucest peut-être ce qu'il y a de plus beau (ucest peut cêre ce quil y a de plus beau. n) Il par tombe à l'endroit de son choix.

La plupart des collègues de Aue ne sont pas des hommes détraqués mais bien ordinaires, époux et hommes detraqués mais bien ordinaires, époux pères, qui digerent mal les ordres horibles de leurs supérieurs. «Schweinerei, c'est une grosse Schweithéorie raciale pseudoscientifique des nazis. Un autre

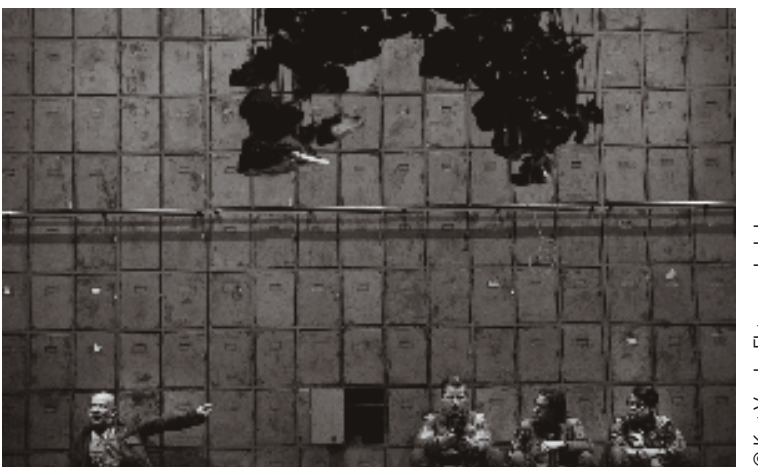

se plaint auprès de Aue : ingénieur, il est formé pour construire des ponts (une «mission culturelle» selo es propres dires), alors qu'en Ukraine il ne s'occupe ene de ports dyna artés.

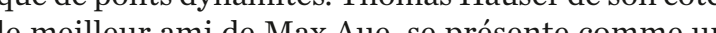

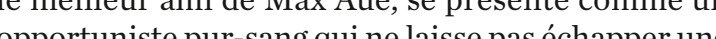
casion de faire carrière.

La décision de mettre l'accent sur la «normalité des officiers provoque une catharsis inverse : le bourreau n'est pa s'ou une par rò distances Le point de dép l'aptation

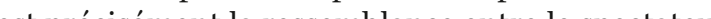
est précisément la ressemblance entre le spectateu et protagoniste. ne sommes-nous pas tous des gen sitution de Aue? Exerciceńnible Lìnoù ag personnage

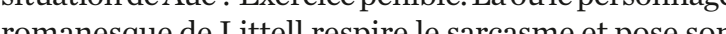
jonesque de Littell respire le sarcasme et pose son jestrice pur étre cocatrice pour être sérieux, Cassiers prend cette thèse on point de vue pourait dépasserunelimite dans conditions varticulières Il s'asser une limite dans des

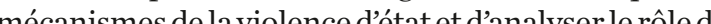
ens gens «ordinaires» dans cette machine, une question qu Browning (Browning 1992) et Harald Welzer (Welzer \&. Christ 2006) depuis des années.

L'identification entre spectateur et protagoniste est imulée par une scénographie réfléchie. Dans l'introductioe par une scenographie réféchie. Dans lintro-

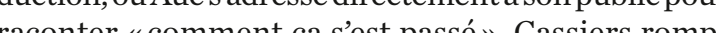
volontairement l'illusion théâtrale. Les lumières dans la salle ne sont pas encore éteintes quand l'Obersturm-

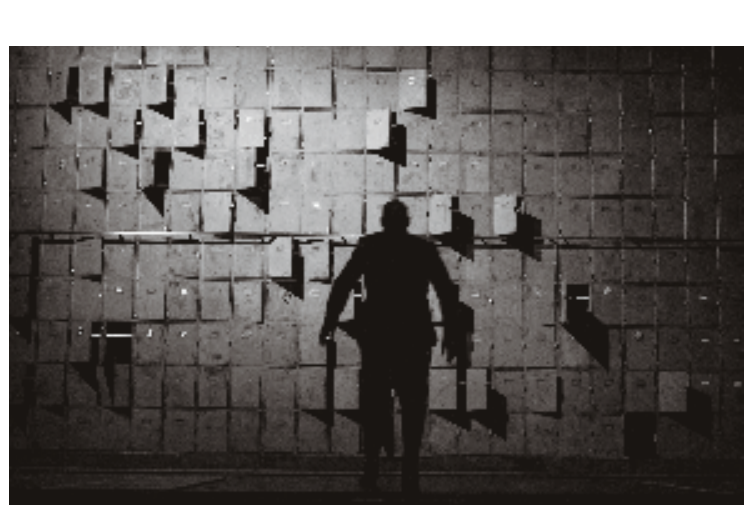


-. bannführer commence son monologue. Elles ne le seront qu’après l'avertissement final de Aue : «Le vra danger pour lhommecestmoi, cest vous. Etsivous n'en es pas ting est peut-être un acteur incarnant un personnage de roman, ce qui se passe sur scène n'est décidénent lité quen du thếtre. Regarder engage la responsabitistan sur astration

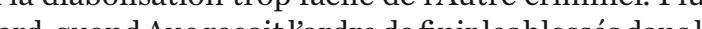
tard, quand Auerȩ̧oit lordrede finir lestessés dans ravin Bar Testing se trouve sur le devant de la scène. De riere Ke lui, un immense écran sur lequel est projeté sa tête, littéreme litéralome fur et à mesure que Aue fait le récit de sa nuit agitée.

Afin de centrer le spectacle sur cet exercice d'idenification, Cassiers et Jans ont dû faire un montage particulier du matériau textuel. Tandis que Littell introduisait le lectenr dans les ariere-chambres obscures de la pensée de son protagoniste - sa relation incestueuse et perverse avec sa sour Una et les de services - le personnage théâtral paraît écé , lepersonnage thếné parait un homm posé et prévenant qui est entrainé par son entourage et sombre dans «un cloaque, un marécage opaque Contrairenent à certains de ces collegues toutefors, ne demande pas à être muté. Par curiosite, il vajusqua " le lui ent tanalyse lui. Assongrand désarrol, ll constate que laversion pour 'horreur suse de soi-même: «on ne sent, à la longue plus grant chose, on pin de rupture qu'il chern Le choche cedo la pla ce a s. toujours plus breve [...], et ainsi, lentement, sans bie 'ene je cherche la lumière. que je cherche la lumière,

LE LANGAGE DU MAL

La pièce Les Bienveillantes s'insère dans une entreprise plus large où Cassiers et Jans explorent le mal en prise plus large oú Cassiers et Jans explorent le mal en tant que mécanisme institutionnel, social et langagie vail de contextualisation historique et d'un projet social.

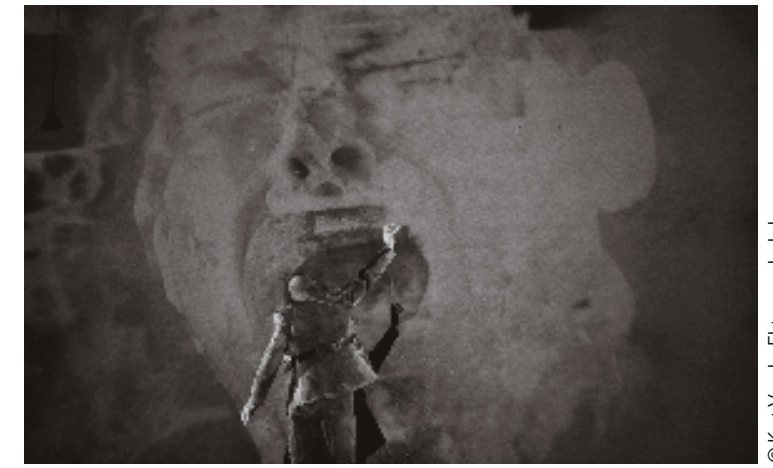

Si le théâtre revendique bel et bien son droit de traiter la Shoah, il ne se passe pas pour autant d'un commentaire historiographique approfondi. Bien au contraire il parait impossible de dépasser le niveau documentaire sui-min fut d'ailleurs loú polle du passé. Littell lui-même fut dailleurs loué pour les recherches en archives qui avaient nourri son roman. Les équipes de la Ton lo en développé le portail Test of Civilisation, un longread en

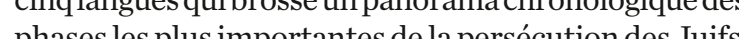
phases les plus importantes de la persécution des Juifs, XIlant des idees antisemites de listorien allemand du XIX siccle, Heinrich von Tretschk, aux déclarations des chefs de file nazis tels qu'Hitler, Himmler et Mengele, et jusqu'à la defense qu'a présentee Eichmann durantsonproces no nazis pour stigmatiser l'autre.

Dans la même ligne, Cassiers a réalisé une étude préliminaire sur base de l'essai Le sec et l'humide de prèliminaire sur base de l'essai Le sec et l'humide de Jonathan Littell. Lauteur y teste les thèses du sociologue allemand Klaus Theleweit portant sur le langage du fasciste belge Léon Degrelle. Le rapport entre pouCoiretlangue estefiectivementundes thèmes favoris de Cassiers. La manipulation du langage peut déconnec-

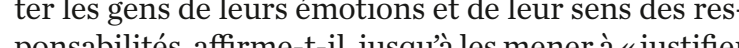
ponsabilités, affirme-t-1, jusqu’a les mener à «justifie linjustifiable» (Perrot \& Cassiers, 2015, p. 67). Dan Aue, qui pèse les conséquences du jargon nazi

On croit encore que les mots désignent des idées. Mais je suis plutôt d'avis qu'il n'y a pas vraiment d'idées. I n'y a réellement que des mots, et le poids propre aux
[Endlösung] et son inévitabilité! Car en vérité comment résister à la séduction d'un tel mot? C'est aussi inconcevable que de résister au mot obéir, au mot servir, au mot loi. Et c'est peut-être là, au fond, la raison pour laquelle, dans notre langage bureaucratique, notre bürokratische Amtsdeutsch, les abstractions et les tournures passive dominent : «il a été décidé », «les Juifs ont été déportés», «cette tâche difficile a été accomplie»... et ainsi les choses se font toutes seules, personne ne fait jamais rien, personne n'agit, des actes sans acteurs.

L’appareil d'état allemand avait inventé un langage qui présentait les choses comme inévitables et néce qui presentait les choses comme inevitables et néces-
saires, et les victimes comme des Untermenschen.

Le jargon nazi comme un écheveau dans leque mpêtrent les gens ordinaires: c'est en cela que réside edanger contre lequel Cassiers veut protéger le spectaveur aujourd hui, enlecontraignantàla réflexion età la viglance. Sur portail Test of Civilisation, Jans meten viontes mécanismé la Shoa violentes dansle passé (lá Shoah en Europe, lApartheid en Afrique du Sud, la ségrégation aux Etats-Unis) et de nos jours (les remarques sexistes, racistes, le hate speech et le harcèlenent). La couverture médiatique delactuellecrise des réfugies, ainsi que la réticence de certains pays européens daccepter des réfugiés, sont conférence d'Evion appelée en 1938 par le pésid conférence d Evian, appelée en 1938 par le président anéricain Roosevest duns lintention délaborer un plan pour laccueil des Juifsfuyantle nazisme. Laplateforme digitale Invisible Cilies, enfin, est le fruit d'une

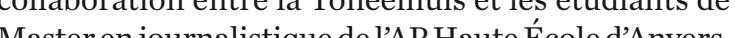
À l'aide de portraits de plusieurs habitants de lanvers. A laide de portraits de plusieurs habitants de la ville confrontés, dunemanière ou dunéautre, à lexclusion, niveau urbain.

Même si la ressemblance entre bourreau et spectateur me semble plus intéressante en tant que question que comme thèse (ne fait-on pas un saut trop important en passant d'une formulation «le nazi n'est pas un constitue icile pointde départ d'un type de théâtre constitue icile point de départ un type de theâtre qui, propre du propre dans le con mant de manière structurelle son engagement social. Anneleen Spiessens
Université de Gand
Plus d'infos

$\Leftrightarrow$ Les Bienveillantes (De Welwillenden) est une adaptation Theâtrale de Guy Cassiers et Erwin Jans. Production:

Bibliographie $\Delta$ Bergman-Carton, Janis (2001): : Christian Boltanskis
Dernières Années: The history of violence and the violence of
history», History \& Memory, vol. 13, $n^{\circ} 1$, p. 3-18. , vol. 13, n 1, p. 3-18

$\Leftrightarrow$ Browning, Christopher (1992): Ordinary Men: Reserve Police Battalion 101
HarperCollins.

\[ \text { Hirsch, Marianne (1996): « Past Lives: Postmemories in } \]
Exile », Poetics Today, vol. 17, n ${ }^{\circ}$ 4, p. 659-686.

$\Leftrightarrow$ Jans, Erwin (2016): : “"Nach Auschwitz ein Gedicht zu schreiben, ist barbarisch" (Adorno): daderperspectief in de
Holocaustrepresentatie», dossier de presse De Welwillenden, Anvers, Toneelhuis

$\diamond$ Littell, Jonathan (2006) : Les Bienveillantes, Paris, Gallimard. $\triangle$ Marsh, Georgia (1990): : «An Interview with Christian

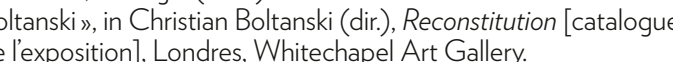
$M$. Mingelgrün, Albert (2009) : «La figure du bourreau naz
au tournant du XXle siècle. Quelques variations littéraires"

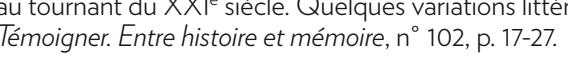

$\diamond$ Perrot, Edwige, et Cassiers, Guy (2015) : « Revisiter P'Histoire et mémoire, $n^{\circ} 121,2015, p .59-63$. Disponible comme récit multimédia (en néerlandais) sur le site web de la Toneelhuis hittp://wwebdoc.toneellhuis.be/deconstructie-van-het-verleden (S)

$\diamond$ Spiessens, Anneleen (2016): Quand l l bourreau prend la

parole: témoignage et fiction, Genève, Droz

$\$$ van Alphen, Ernst (2007): « Deconstructing the Archive:
Visual Archives as Preposterous History», Art History, vol.30, no 3 , p. 364-382

$\diamond$ Welzer, Harald et Christ, Michaela (2006): Täter: Wie aus ganz normalen Menschen Massenmörder werden, Frankfurt am

\section{Sites web}

$\diamond$ Invisible Cities, http://invisible-cities.eu
consulté le 30 mars 2016).

$\diamond$ Test of Civilisation, http://testoffivilisation.eu
(consulté le 30 mars 2016). 